\title{
Tratamento cirúrgico de fraturas osteoporóticas múltiplas da coluna dorsolombar: Relato de caso*
}

\section{Surgical Treatment of Multiple Osteoporotic Fractures of the Dorsolumbar Spine: Case Report*}

\author{
Ricardo Ramalho Marques ${ }^{10}$ Diogo Lino Moura1(i) Paulo Lourenço ${ }^{1(1)}$ \\ ${ }^{1}$ Serviço de Ortopedia e Traumatologia, Centro Hospitalar e \\ Universitário de Coimbra, Portugal \\ Rev Bras Ortop 2021;56(2):258-262. \\ Endereço para correspondência Ricardo Ramalho Marques, Serviço \\ de Ortopedia e Traumatologia, Centro Hospitalar e Universitário de \\ Coimbra, Praça Mota Pinto, 3000-075, Coimbra, Portugual \\ (e-mail: rmmarqueso@gmail.com).
}

\section{Resumo \\ Palavras-chave \\ - osteoporose \\ - fraturas ósseas \\ - cifoplastia \\ - fixação de interna de fraturas}
Abstract
Keywords
- osteoporosis
- fractures, bone
- kyphoplasty
- fracture fixation, internal

As fraturas vertebrais osteoporóticas são um tipo comum de fratura e afetam um número significativo da população com osteoporose. Apesar do elevado risco de fratura, a ocorrência concomitante de fraturas vertebrais em níveis não contíguos é muito rara. Reportamos o caso de uma paciente com três fraturas explosivas da coluna dorsolombar em níveis não contíguos, tratada com cifoplastia e fixação posterior transpedicular por via percutânea. Seis meses após a cirurgia, a paciente tem marcha autônoma, sem dor, e, radiologicamente, não existem evidências de perda de redução das fraturas.

Osteoporotic vertebral fractures are common and affect a significant number of subjects with osteoporosis. Despite the high fracture risk, the concomitant occurrence of vertebral fractures at non-contiguous levels is very rare. We report the case of a patient with three burst dorsolumbar spine fractures at non-contiguous levels who was treated with percutaneous kyphoplasty and transpedicular posterior fixation. Six months after the surgery, the patient walks autonomously and without pain; in addition, there is no radiological evidence of fracture reduction loss.

\section{Introdução}

As fraturas vertebrais em compressão do tipo A da classificação $A O$ Spine são uma das consequências clínicas mais relevantes da osteoporose e cuja incidência tem aumentado

\footnotetext{
Trabalho desenvolvido no Serviço de Ortopedia e Traumatologia, Centro Hospitalar e Universitário de Coimbra, Portugal.
}

recebido

25 de Maio de 2020

aceito

17 de Setembro de 2020

Publicado online

Março 22, 2021 na população mais idosa. ${ }^{1}$ São potencialmente causadoras de dor crônica, com limitação funcional e impacto significativo nas atividades físicas, qualidade de vida e mortalidade. As fraturas podem ocorrer espontaneamente ou devido a um evento traumático, geralmente por mecanismo compressivo envolvendo o corpo vertebral e, mais frequentemente, envolvem o terço médio da coluna torácica e a região dorsolombar.

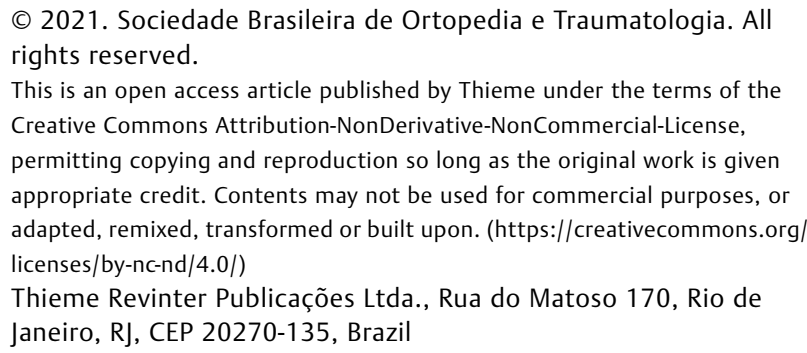

This is an open access article published by Thieme under the terms of the Creative Commons Attribution-NonDerivative-NonCommercial-License, permitting copying and reproduction so long as the original work is given appropriate credit. Contents may not be used for commercial purposes, or adapted, remixed, transformed or built upon. (https://creativecommons.org/ licenses/by-nc-nd/4.0/) Thieme Revinter Publicações Ltda., Rua do Matoso 170, Rio de Janeiro, RJ, CEP 20270-135, Brazil 

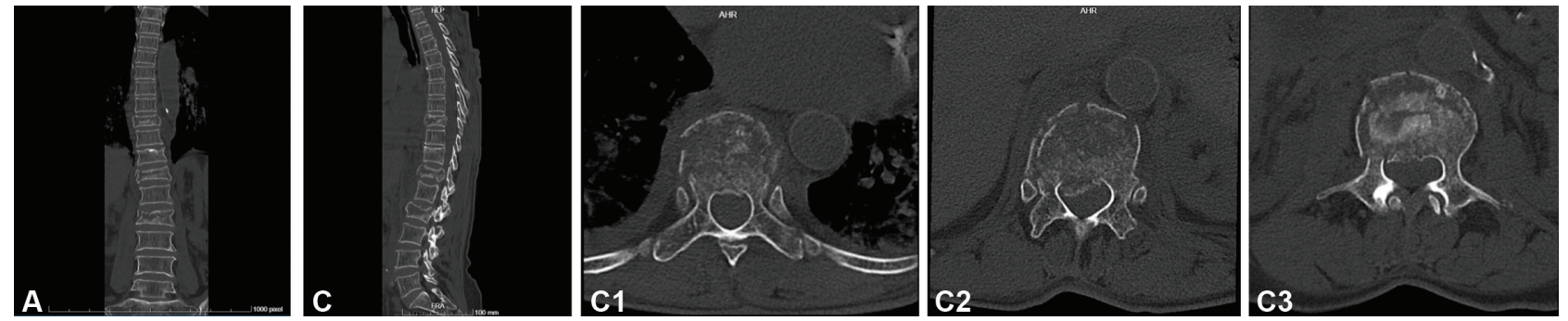

Fig. 1 Planos coronal (A), sagital (B) e axial (C) de tomografias computadorizadas com fraturas explosivas de D9, D12 e L2 numa paciente do sexo feminino, de 70 anos de idade, que condicionam retropulsão ligeira dos muros posteriores.

Embora a maioria destas fraturas sejam estáveis e possam ser tratadas conservadoramente através de repouso, analgesia e ortóteses imobilizadoras, existe um número considerável de fraturas, particularmente na transição dorsolombar que podem, progressivamente, causar perda da altura, aumentar a deformidade em cifose, ou outras, podendo até por rotura do muro posterior provocar compressão nervosa e déficits neurológicos, e justificar estabilização cirúrgica ad initium. Contudo, trabalhos publicados mostram elevadas taxas de complicações em pacientes com osteoporose submetidos a tratamento cirúrgico por cifoplastia, instrumentação posterior, entre outros, com fraturas vertebrais adjacentes e falência de material a afetarem cerca de um terço destes pacientes, em algumas séries. ${ }^{2}$

\section{Descrição do caso}

Reportamos o caso de uma paciente muito ativa, de 70 anos de idade, com osteoporose, do sexo feminino, que sofreu queda da própria altura no domicílio após lipotimia, apresentando de imediato dor na transição dorsolombar. Após 3 dias de dor intensa e persistente, apesar da medicação antiinflamatória e analgésica, recorreu ao hospital. Tinha dor à palpação das apófises espinhosas da transição dorsolombar, sem déficit de força muscular dos membros inferiores e reflexos osteotendinosos normais. Foram diagnosticadas fraturas do tipo A4, segundo a classificação AO Spine, dos corpos vertebrais de D9, D12 e L2, em contexto de osso osteoporótico (- Figura 1), numa paciente que não tinha patologia associada nem tomava cronicamente medicação predisponente à osteoporose. As fraturas apresentavam sinal de esmagamento importante das trabéculas ósseas intrassomáticas.

A paciente foi submetida ao tratamento cirúrgico 4 dias após admissão hospitalar (-Figura 2). Foram efetuadas cifoplastias em D9, D12 e L2 com aplicação de stents intrassomáticos por acesso percutâneo transpedicular e preenchimento com polimetilmetacrilato (PMMA) de alta viscosidade. Por incapacidade do corpo vertebral de D9

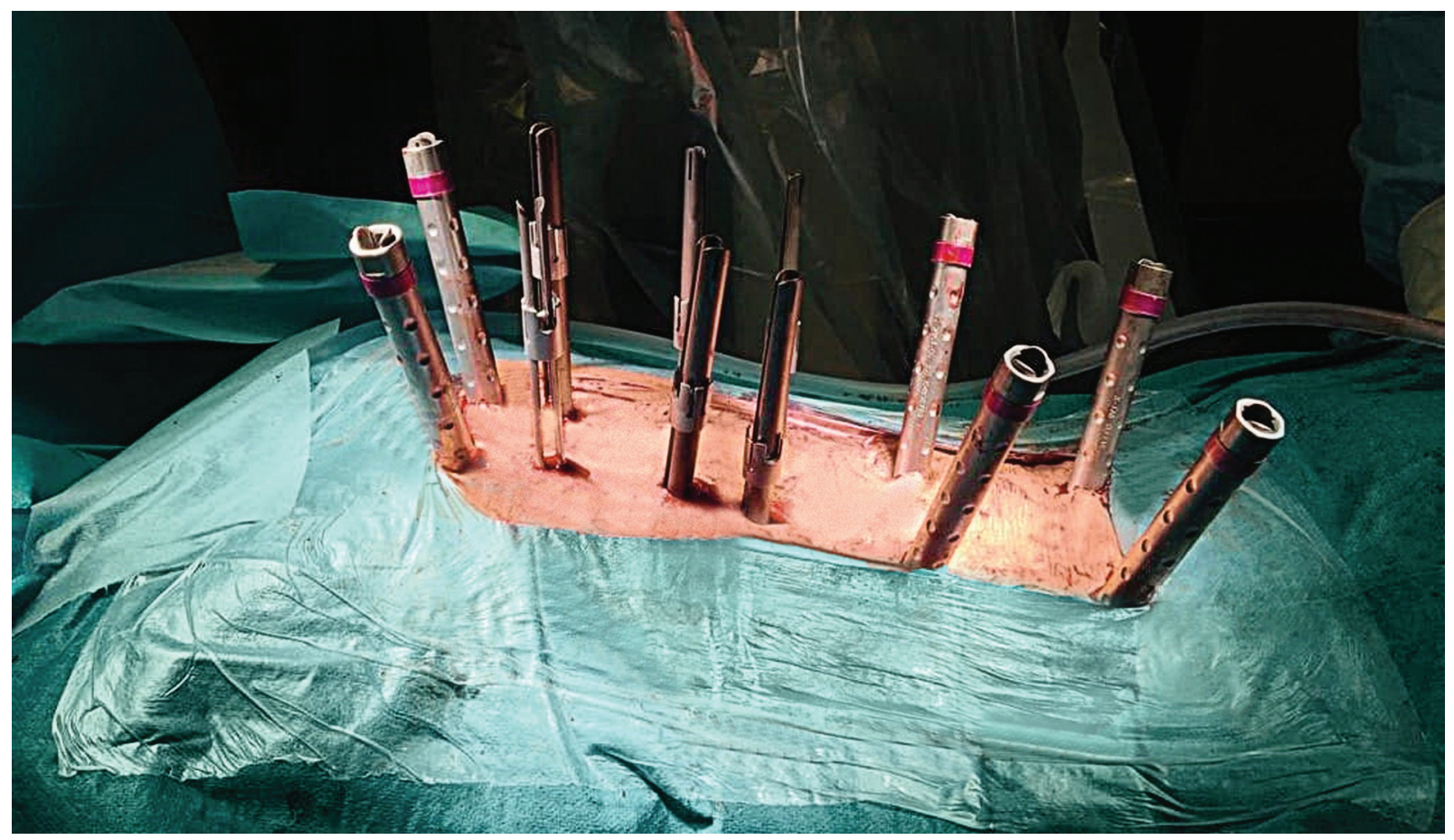

Fig. 2 Aplicação da instrumentação posterior por via percutânea para fixação transpedicular D7, D8, D10, D11, L1 e L3 após realização das cifoplastias em D9, D12 e L2 (A). 

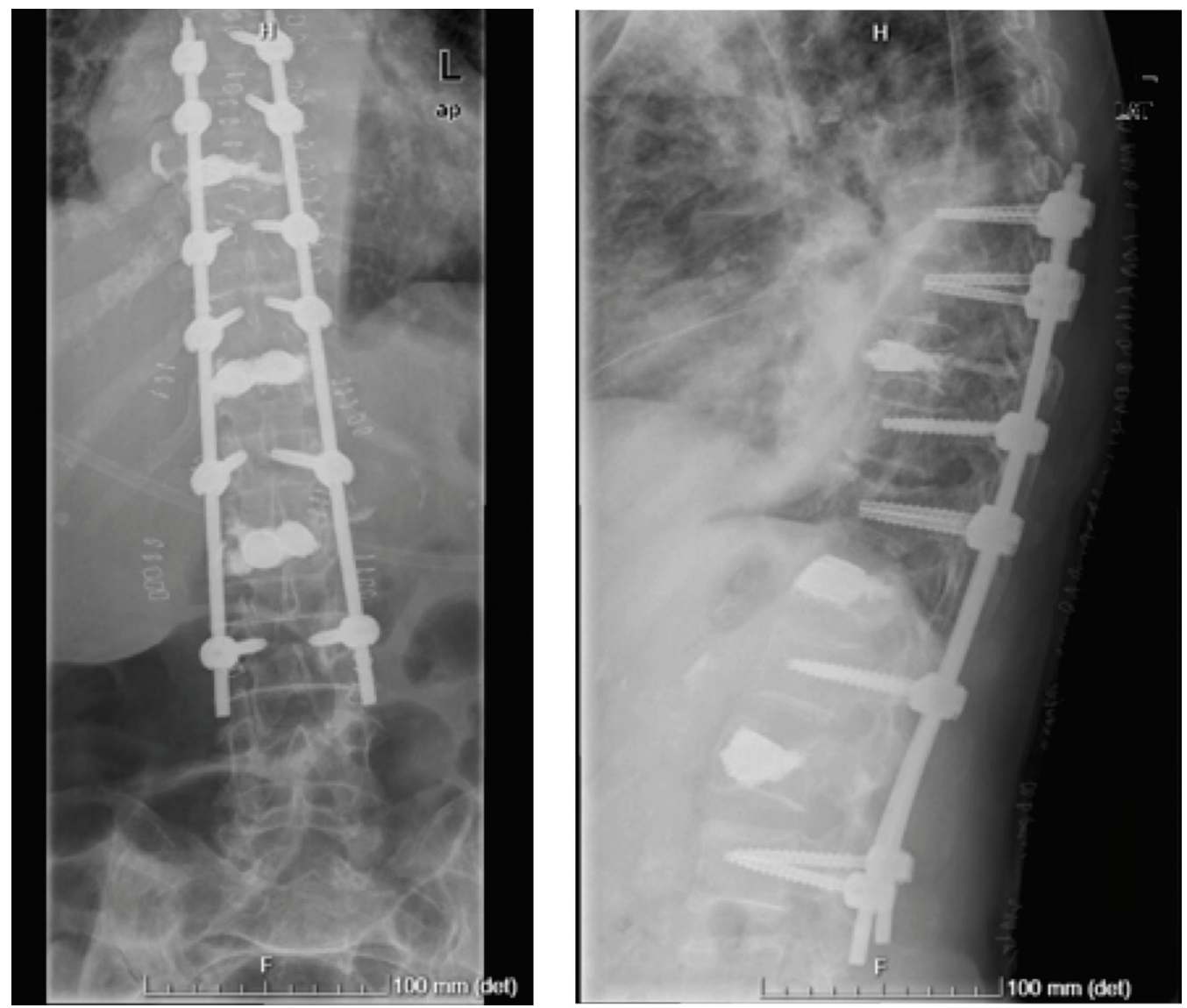

Fig. 3 Radiografia pós-operatória da fixação posterior transpedicular D7, D8, D10, D11, L1 e L3, após restauração da altura das vértebras fraturadas D9, D12 e L2 por cifoplastia.

incluir 2 stents, mesmo do tamanho mais reduzido (small), optou-se pela aplicação de um stent único neste nível e preenchimento com cimento. Realizou-se uma fixação posterior transpedicular percutânea das vértebras D7-D8-D10D11-L1-L3 com aplicação de barras moldadas de acordo com a anatomia da paciente, em cifose torácica e lordose lombar (sistema VIPER, DePuy/Synthes, Warsaw, IN, EUA) (-Figura 3).

A paciente conseguiu se levantar no primeiro dia do pósoperatório e, posteriormente, caminhou com dorsolombostato. Às 6 semanas de pós-operatório, a marcha era autônoma, apenas com dor residual. Iniciou tratamento antiosteoporótico com denosumab, e aos 6 meses de pósoperatório deambula autonomamente, sem queixas álgicas ou perda de redução das fraturas, e com evolução cicatricial favorável (- Figura 4).

\section{Discussão}

A paciente discutida no presente artigo apresenta um raro caso de fraturas osteoporóticas de vários níveis não contíguos da coluna dorsolombar, tratado de forma percutânea, em que se garantiu não só suporte anterior pelas cifoplastias, mas também suporte da banda de tensão posterior através da fixação posterior. A opção, nesta paciente, é justificada pelo elevado risco de desenvolver cifose progressiva. Não obstante, a instrumentação tem uma extensão considerável, e a extração poderá ser ponderada após a consolidação das fraturas.

Um dos principais riscos das fraturas osteoporóticas vertebrais é a ocorrência progressiva do colapso do corpo vertebral, assim como o desenvolvimento gradual de

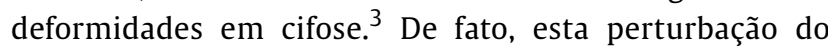
equilíbrio sagital adquire maior relevo nos doentes idosos com osteoporose, uma vez que a probabilidade de agravamento progressivo das alterações sagitais da coluna é maior. Consequentemente, a tensão dos músculos paravertebrais aumenta, provocando dor crônica, e o desequilíbrio sagital pode inclusivamente causar novas fraturas. Além disso, um número de fraturas vertebrais mais elevado deslocará mais ainda o centro de gravidade da coluna anteriormente.

A cifoplastia com PMMA é uma abordagem bem documentada na correção e na prevenção do colapso e deformidades, importante no restauro da harmonia e equilíbrio sagital global da coluna. ${ }^{4}$ No entanto, a correção obtida isoladamente por este tipo de procedimento pode, em $10 \mathrm{a}$ $30 \%$ dos casos, falir gradualmente e causar aumento da cifose. ${ }^{5}$ Por outro lado, até $25 \%$ dos casos desenvolve fraturas em nível adjacente, frequentemente a vértebra osteoporótica superior. $^{3}$

No caso apresentado, a presença de 3 fraturas explosivas concomitantes em D9, D12 e L2, com elevado risco de colapso pós-traumático, aumenta consideravelmente o risco de 

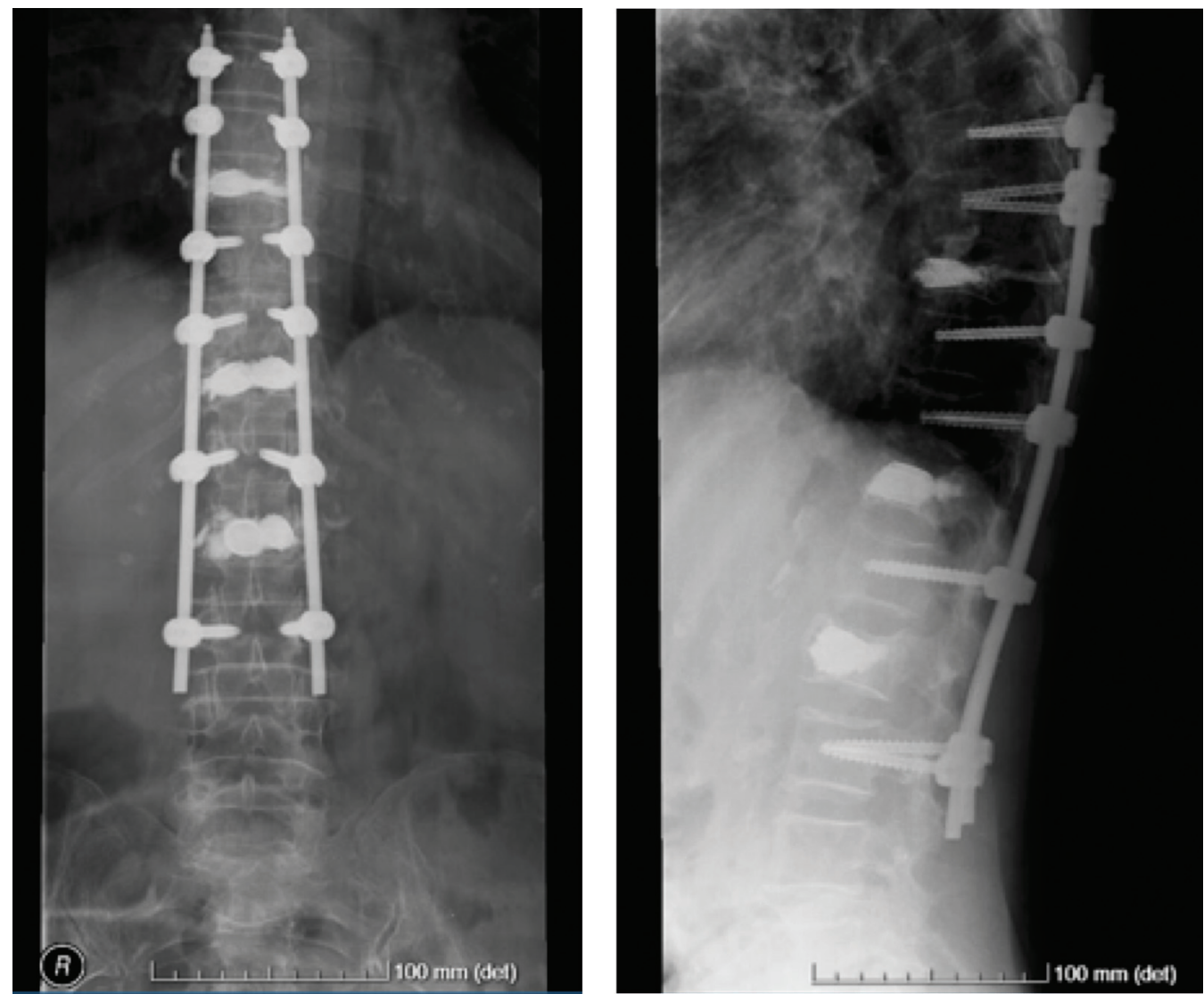

Fig. 4 Controle radiológico 6 meses após a cirurgia.

complicações da fixação posterior isolada. A força de cada ponto de fixação da instrumentação posterior é menor na coluna osteoporótica, uma vez que a força de pullout, o torque de cutout e o torque máximo de inserção são diretamente proporcionais à densidade mineral óssea. ${ }^{6} \mathrm{~A}$ presença de osteoporose em pacientes submetidos a tratamento cirúrgico da coluna tem sido associada com fraturas vertebrais após instrumentação, pseudartroses e à falência secundária de material. ${ }^{7}$ Estudos biomecânicos têm demonstrado que o suporte insuficiente da coluna anterior pode ser responsável por estes resultados insatisfatórios, além da fixação precária que o osso de baixa densidade mineral oferece. ${ }^{8}$ Além do mais, a cavidade formada no corpo vertebral fraturado após a distração atrasa a consolidação e favorece a perda de redução. ${ }^{9}$ Optou-se, assim, por combinar a cifoplastia com stent e a fixação posterior percutânea. Decidiu-se aplicar stents na medida em que estes implantes além de oferecerem uma maior rigidez do corpo vertebral, permitem diminuir o risco de extravasamento de cimento porque criam uma cavidade intrassomática rodeada pelo implante. Nas fraturas em compressão, esta técnica híbrida foi associada com menos dor no período pós-operatório imediato e possibilitou a manutenção da altura do corpo vertebral fraturado praticamente total, com um baixo risco de recorrência da cifose. ${ }^{10}$ Desta forma, acreditamos mitigar o risco de colapso anterior por ausência de suporte na coluna anterior na fixação posterior, assim como o risco de falência do material de fixação e das fraturas osteoporóticas adjacentes.

Conflito de interesses

Os autores declaram não haver conflito de interesses.

\section{Referências}

1 Johnell O, Kanis JA. An estimate of the worldwide prevalence and disability associated with osteoporotic fractures. Osteoporos Int 2006;17(12):1726-1733

2 Goldstein CL, Brodke DS, Choma TJ. Surgical Management of Spinal Conditions in the Elderly Osteoporotic Spine. Neurosurgery 2015;77(Suppl 4):S98-S107

3 Frankel BM, Monroe T, Wang C. Percutaneous vertebral augmentation: an elevation in adjacent-level fracture risk in kyphoplasty as compared with vertebroplasty. Spine J 2007;7(05):575-582

4 Papanastassiou ID, Phillips FM, Van Meirhaeghe J, et al. Comparing effects of kyphoplasty, vertebroplasty, and non-surgical management in a systematic review of randomized and nonrandomized controlled studies. Eur Spine J 2012;21(09): 1826-1843

5 Komemushi A, Tanigawa N, Kariya S, et al. Percutaneous vertebroplasty for osteoporotic compression fracture: multivariate study of predictors of new vertebral body fracture. Cardiovasc Intervent Radiol 2006;29(04):580-585

6 Paxinos O, Tsitsopoulos PP, Zindrick MR, et al. Evaluation of pullout strength and failure mechanism of posterior instrumentation in normal and osteopenic thoracic vertebrae. J Neurosurg Spine 2010;13(04):469-476 
262 Tratamento cirúrgico de fraturas osteoporóticas múltiplas da coluna dorsolombar Marques et al.

7 DeWald CJ, Stanley T. Instrumentation-related complications of multilevel fusions for adult spinal deformity patients over age 65: surgical considerations and treatment options in patients with poor bone quality. Spine (Phila Pa 1976) 2006;31(19 suppl): S144-S151

8 Norton RP, Milne EL, Kaimrajh DN, Eismont FJ, Latta LL, Williams SK. Biomechanical analysis of four- versus six-screw constructs for shortsegment pedicle screw and rod instrumentation of unstable thoracolumbar fractures. Spine J 2014;14(08):1734-1739
9 Knop C, Fabian HF, Bastian L, Blauth M. Late results of thoracolumbar fractures after posterior instrumentation and transpedicular bone grafting. Spine 2001;26(01): 88-99

10 Zhang J, Liu H, Liu $\mathrm{H}$, et al. Intermediate screws or kyphoplasty: Which method of posterior short-segment fixation is better for treating single-level thoracolumbar burst fractures? Eur Spine J 2019;28(03):502-510 